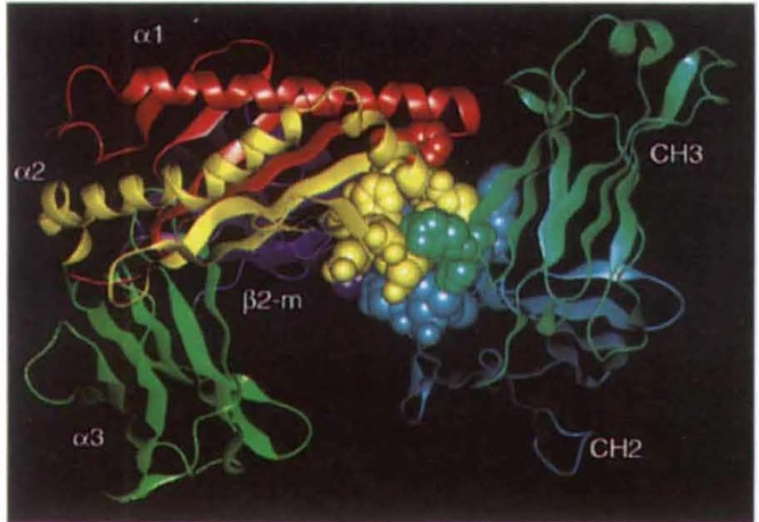

FIG. 2 Principal interactions between the FcRn and Fc, illustrated by ribbon diagrams of FcRn (domains $\alpha 1, \alpha 2, \alpha 3$ and $\beta 2 \mathrm{~m}$ are in red, yellow, green and purple, respectively) and of $\mathrm{Fc}\left(\mathrm{CH}_{2}\right.$ and $\mathrm{CH} 3$ domains in blue and light green). The main contact residues ( $\alpha 1$ domain, 90; $\alpha 2,113-119$ and $131-135 ; \beta 2 \mathrm{~m}, 1-4$ and 86 ) are depicted as space-filling structures. Contact residues from $\alpha 3 \mathrm{FcRn}$ dimer to Fc are not shown. (Details taken from ref. 2 .)

all MHC molecules studied, does not copurify with bound peptide.

The structure of the co-crystal of FcRn complexed with $\mathrm{Fc}$, at $\mathrm{pH} 6.5$, reveals the main site of contact between the two molecules. This is at the edge of the $\alpha 1 / \alpha 2$ domain region, a region distinct from the sites of interaction with peptide, CD8 or T-cell receptor. This part of the Fcbinding site of the FcRn (Fig. 2) is formed by three $\beta$-turns of $\alpha 1 / \alpha 2$, lying to the side where the carboxy-terminal portion of bound peptide resides in a classic class I molecule. Additional contributions to Fc binding derive from the amino terminus of $\beta 2 \mathrm{~m}$ and a loop from that domain. The surface of the Fc that interacts with the FcRn derives from both the $\mathrm{CH} 2$ and $\mathrm{CH} 3$ domains, and probably exploits three or four titratable histidines as contacts. Both the isolated FcRn and the complex with Fc reveal higher order dimers, formed by juxtaposition of the Ig-like domains of the $\mathrm{FcRn}$. The reason for such dimerization is unknown, but does offer a compelling model for the interaction with IgG.

So a new functional role has been adopted by using yet another surface of this versatile molecule. With the available evolutionary comparisons, it is impossible to predict if the common ancestor of MHC and FcRn had a functional groove which was lost by $\mathrm{FcRn}$ after divergence of the molecules, or if MHC molecules acquired a functional groove driven by their need to display self- or antigenic peptides for presentation to $T$ cells. Further evolutionary comparisons certainly seem warranted. Moreover, although both structural and biochemical data strongly support the notion that this MHC-like molecule does not employ its potential groove to bind peptide, the question remains whether this site might interact with other ligands. The demonstration ${ }^{8}$ that $\mathrm{CD} 1 \mathrm{~b}$, a non-polymorphic, $\beta 2 \mathrm{~m}$-associated mem- ber of the MHC family binds a lipid antigen, mycolic acid, and presents it to $\mathrm{T}$ cells with $\alpha \beta$ T-cell receptors, raises the possibility that FcRn may also play a role in presentation of nonpeptide antigens.

How general might this first, three-dimensional structure of an $\mathrm{Fc}$ receptor be? Two lines of evidence suggest that it may well tell us something about other FcRs of the immunoglobulin domain family. First, modelling of the IgE highaffinity $\mathrm{FcR}, \quad \mathrm{F} \in \mathrm{RI}$ based on deletion studies, sequence comparisons with CD4 and $\mathrm{CD} 2$, and estimates of the distance from the amino to the carboxy terminus of $\operatorname{IgE}$ - indicate a 'lying down' structure, where the long axis of the molecule sits parallel to the membrane ${ }^{9}$, allowing a dimer interaction similar to that observed in the FcRn structure. Second, the greatest sequence similarities between FcRn and other FcRs lie in the $\alpha 3, \mathrm{Ig}$-like domain, which may contact the $\mathrm{CH} 2$ domain in the dimer predicted by this model. The predictive value of such modelling will be readily testable by mutational mapping of potential IgG-binding sites.

Each unique member of the Fc receptor family has attracted considerable attention for its importance in mediating various aspects of the immune response, ranging from lymphocyte modulation to inflammation and immunoglobulin transport. Given the surprises to be found in the three new papers ${ }^{1-3}$, further threedimensional structures of each of these molecules, and of their complexes with $\mathrm{Fc}$, will be eagerly awaited.

Jeffrey V. Ravetch is in the Molecular Biology Program, Memorial Sloan-Kettering Cancer Center, 1275 York Avenue, New York, New York 10021, USA. David H. Margulies is in the Laboratory of Immunology, National Institute of Allergy and Infectious Diseases, National Institutes of Health, Bethesda, Maryland 20892, USA.

1. Burmeister, W. P., Gastinel, L. N., Simister, N. E., Blum, M. L. \& Bjorkman, P. J. Nature 372, 336-343 (1994)

2. Burmeister, W. P., Huber, A. H. \& Bjorkman, P. J. Nature 372, 379-383 (1994)

3. Story, C. M., Mikulska, J. E. \& Simister, N. E. J. exp. Med. 180. 2377-2381 (1994)

4. Ravetch, J. V. Cell 78,553-560 (1994)

5. Simister, N. E. \& Mostov, K. E. Nature 337, 184-187 (1989).

6. Rodewaid, R.J. Cell Biol. 71, 666-670 (1976)

7. Roberts, D. M. Guenthert, M. \& Rodewald, R. J. Cell Biol. 111, 1867-1876 (1990)

8. Beckman, E. M. et al. Nature (in the press)

9. Beavil, A. J. et al. Biochem. Soc. Trans. 21, 968-972 (1993).

10. Fremont, D. H. et al. Science 257, 919-927 (1992)

\section{Free the spirit!}

LAST week, Daedalus was expounding the physics of poltergeists. The spirit world, he reckoned, coexists in the same space as the material one, but is much colder: perhaps at the cosmic background $3 \mathrm{~K}$. A poltergeist works by abstracting radiant heat from the material world and letting it run down into the spiritual world. It is simply a heat engine.

He is now risking a more teleological interpretation. The common theory of ghosts, he points out, is that they are the souls of dead people, disconsolately haunting the region where they died. Only a few unlucky souls seem to get stuck in this way. Possibly such a soul fails to carry away sufficient animal heat from its previous abode: in the cold spiritual world it is simply frozen to the spot. To free itself for further adventures, it needs to acquire enough latent heat of evaporation. Haunting is simply its attempt to steal heat from the material world. This is why it causes cooling. The disturbance and noise may be pure waste motion, a sort of counterpart to waste heat. Alternatively, they may be intended to stir up helpful emotions in the human audience. A human mind is, after all, a link between the two worlds, and may be essential to the heat transfer process.

This theory has several implications. It suggests that cremation, which gives the soul the heat it needs to fly free from the body, is the best way of disposing of the dead. This may have been sensed by those ancient cultures who burnt their nobles on heroic funeral pyres. It implies that a dying individual should undergo a sharp drop in temperature, as his departing soul leaves for the spirit world and takes a little heat with it. It further suggests that, in the traditional exorcism ritual with bell, book and candle, it is the candle that does most of the work. It provides the necessary radiant energy.

But radiant energy from a candle flame at some $2,000 \mathrm{~K}$ is badly matched to a sink at $3 \mathrm{~K}$. A truly efficient exorcism would warm the frozen ghost with matching $3 \mathrm{~K}$ 'cosmic background' microwave energy. The radiation from a standard microwave oven would probably do very well. Furthermore, its hundreds of watts of power would be far more effective than any candle. At first Daedalus feared that the oven would need to be rigged to work with its door open, to flood the room with microwaves. But he now reckons that the ghost could pass through the oven wall to access the inside directly. Bell, book and microwave oven would free the trapped ghost to roam the spirit world, and free its human hosts from its haunting, chilling pleas for release.

David Jones 